\title{
A Power Efficiency Wireless Communication Networks by Early Detection of Wrong Decision Probability in Handover Traffic
}

\author{
Patil Shweta $\mathbb{D}^{1},{ }^{1}$ Leena Bojaraj, ${ }^{2}$ Pallavi Biradar, ${ }^{3}$ Mohammed Bakhar, ${ }^{4}$ \\ and Alazar Yeshitla ${ }^{5}$ \\ ${ }^{1}$ Guru Nanak Dev Engineering College, Mailor Road, Bidar, India \\ ${ }^{2}$ Department of Electronics and Communication Engineering, KGiSL Institute of Technology, Coimbatore, India \\ ${ }^{3}$ Department of Electronics and Communication Engineering (RङD), Guru Nanak Dev Engineering College, Bidar, India \\ ${ }^{4}$ Electronics and Communication Engineering, Guru Nanak Dev Engineering College, Bidar, India \\ ${ }^{5}$ Department of Biotechnology College of Biological and Chemical Engineering, Addis Ababa Science and \\ Technology University, Ethiopia
}

Correspondence should be addressed to Patil Shweta; shweta.gadgay@gmail.com and Alazar Yeshitla; alazar.yeshi@aastu.edu.et Received 27 December 2021; Revised 13 January 2022; Accepted 15 January 2022; Published 18 February 2022

Academic Editor: Deepak Kumar Jain

Copyright (c) 2022 Patil Shweta et al. This is an open access article distributed under the Creative Commons Attribution License, which permits unrestricted use, distribution, and reproduction in any medium, provided the original work is properly cited.

\begin{abstract}
This paper highlights the energy consumption due to handovers in wireless communication system. Ever-increasing demand for higher data rate for users of cellular networks and the energy consumption that results from the effective satisfaction of these demands leads to greater consumption of energy from such energy sources that depend on burning of greenhouse gas emitting fossil fuels for energy production. An evolved Node B or e-NB inside, as in equipment handling the radio frequencies, can be divided into two large categories (1) BaseBand Unit (2) Remote Radio Head or RRH. For the purpose of our current work, we will focus on mainly the Remote Radio Head part. A simple picturization of the evolved Node B and the User Equipment (UE) that are included in the power model has been discussed. The proposed framework for mobility management/security gateway protocol including wrong decision probability of handover evaluation following measurements' procedure has been designed successfully. We have compared to other UL/UE power consumption. The lowest ISD has the largest power usage in resultant output.
\end{abstract}

\section{Introduction}

A seamless context-aware architecture for fourth generation wireless networks, Handoffs in fourth generation heterogeneous networks, is designed [1]. Nowadays, the mobile wireless network evolution and emerging difficulties is also highlighted in communication [2]. Deng et al. proposed a Quality of Service (QoS) system for multimedia transmission using IEEE 802.11 wireless LANs. Comparison of vertical handoff decision algorithms for heterogeneous wireless networks and vertical handoff method for cellular multihop networks which has been reviewed from the vertical handoff decision technique is presented [3-5]. In the wireless overlay networks is an adaptive technique for a vertical handoff method. Parallel and distributed dystems are two types of systems that can be used together.
Optimizations for vertical handoff decision algorithms were developed by Zhu et al. in 2004 [6-8]. In the upcoming year, wireless networks will use active application-oriented vertical handoff technique. With the heterogeneous networks, handover decision with fuzzy MADM has been explained [9-11]. The Wireless Communications and Networking Conference is a gathering of people who are interested in wireless communications and networking. For next-generation networks, a network selection process is in place [12-14]. The wireless overlay network is an adaptive technique for a vertical handoff method. The large bandwidth industrial IoT has advantages due to positioning-assisted communication technology involved [15-18]. Future air traffic control communications will require the design of airground data linkages [19-24]. Architectural difficulties and potential for air-ground integrated mobile edge networks. A 
method for new computing method on the Internet of Things that integrates the air and ground as per survey of the integrated space-air-ground network [25-34].

\section{Background}

Ever-increasing demand for higher data rate from users of cellular networks and the energy consumption that results from successful satisfaction of these demands leads to greater consumption of energy from such energy sources that depend on burning of greenhouse gas emitting fossil fuels for energy production. In [1], the authors have highlighted that, in India, telecommunication system consumes about 2.5 billion liters of diesel at the cost of 1.4 billion U.S. dollars that causes emissions of about 5 billion tons of $\mathrm{CO}_{2}$ annually. A significant amount of energy is lost during the process of call drops that occur largely because of inaccurate handover processes. Apart from advising mobile operators to adopt inexpensive, spotless, dependable, and sustainable power sources, the telecommunication research community can provide procedures to avoid call drops by the process of avoiding wrong handovers. In the literature, a lot of research studies have been proposed that aim to minimize the power loss due to handovers in wireless cellular networks. In the following sections, we would give a brief review of the research work that has been carried out on the topics of energy consumption related to the handover processes in wireless cellular networks. In [2], the basic material which gives a detailed explanation of the procedure of handover procedure is provided. In [3], Song et al. propose a vertical handover scheme that tries to minimize the power that is consumed during the handover procedure. In [4], Tayyab et al. explored the measurement of power consumption in LTE networks during handover procedures.

In another paper by Ghaderi and Boutaba [5], a new methodology of evaluating power consumption in LTE networks during the handover process from the network controller has been proposed, which provides an easier technique of measuring power consumed in LTE networks during handover procedures.

In [6], Tuysuz et al. presented a survey of the different algorithms and procedures that have been developed by multiple researchers for the implementation of processes of vertical handovers in long-term evolution networks or LTE networks that are efficient in terms of energy consumption during the process of handover from one network node to another network node during the movement of the user equipment across geographical distances.

The procedure of handover in 4G-LTE can be classified into different stages, namely, handover preparation (HP) andhHandover execution (HE). These stages have been described in detail in Figure 1. In Figure 1, we present the procedures that have been proposed for the management of mobility and the security gateway.

The user equipment measures and identifies which of the nearby cells will be best for it for the process of transmission of signals. After this, the user equipment sends a report to the evolved Node B or e-NB that is currently serving it. Basically, the user equipment performs a measuring of signal powers by sending a reference signal and measuring the received power from both the current e-NB and also from adjacent e-NBs. After these measurements have been completed a measurement report is generated that is transferred to the e-NB that is currently serving it. During the transferring of this measurement report, it is checked whether the received power from the reference signal is of higher quality than the signals received from the currently serving e-NB. Figure 2 shows mobility management entity and security gateway handover procedure.

If it is found that the signal quality from the adjacent $\mathrm{e}-\mathrm{NB}$ is higher than that received from the e-NB that is currently serving the user equipment, then preparation of the handover process from the serving e-NB to the adjacent $\mathrm{e}-\mathrm{NB}$ is initiated.

This process takes place in the following steps:

(1) A formal request is sent to the neighboring e-NB from the currently serving e-NB to initiate the handover procedure

(2) After this, a command is generated that is transferred to the concerned user eEquipment from the serving e-NB to get prepared for the handover process

(3) Following this, the concerned user equipment transfers the messages to the newly targeted e-NB

(4) After the reception of this message from the user equipment, the targeted e-NB is sent a handover confirmation message through the transmission of a random access channel transmission

(5) After this, the targeted e-NB sends a confirmation message to the previous supporting e-NB about the success of the handover process

It is at this point we want to bring in our innovation regarding the transmission of the handover message.

\section{Proposed Methodology}

3.1. Model of Energy Consumption between the User Equipment and $e-N B$. Inside an evolved node $\mathrm{B}$ or e-NB, as in [8], equipment handling the radio frequencies can be divided into two large categories: (1) BaseBand Unit and (2) Remote Radio Head or RRH. For the purpose of our current work, we will focus on mainly the Remote Radio Head part. An illustration of the considered engagement between the e-NB and the User Equipment has been presented in Figure 1.

In Figure 1, we represent $P_{x \text {,sup }}$ as the supply power to generate the signal output power $E_{x}$, where $x=\{e \mathrm{NB}, \mathrm{UE}\}$. In the current problem, we are mainly interested about the contribution of the handover procedure to $E_{x \text {,sup }}$.

The power distribution algorithm for both the e-NB and the user equipment (UE) will fine-tune the power level that has been allocated for every subcarrier and resource segments:

$$
\begin{aligned}
E_{e_{-} \mathrm{NB}}^{\mathrm{RB}} & =P_{e \mathrm{NB}} \wedge M_{\mathrm{RB}}^{\mathrm{DL}}, \\
E_{\mathrm{UE}}^{\mathrm{RB}} & =\frac{P_{\mathrm{UE}}}{M_{\mathrm{RB}}^{\mathrm{DL}}}
\end{aligned}
$$



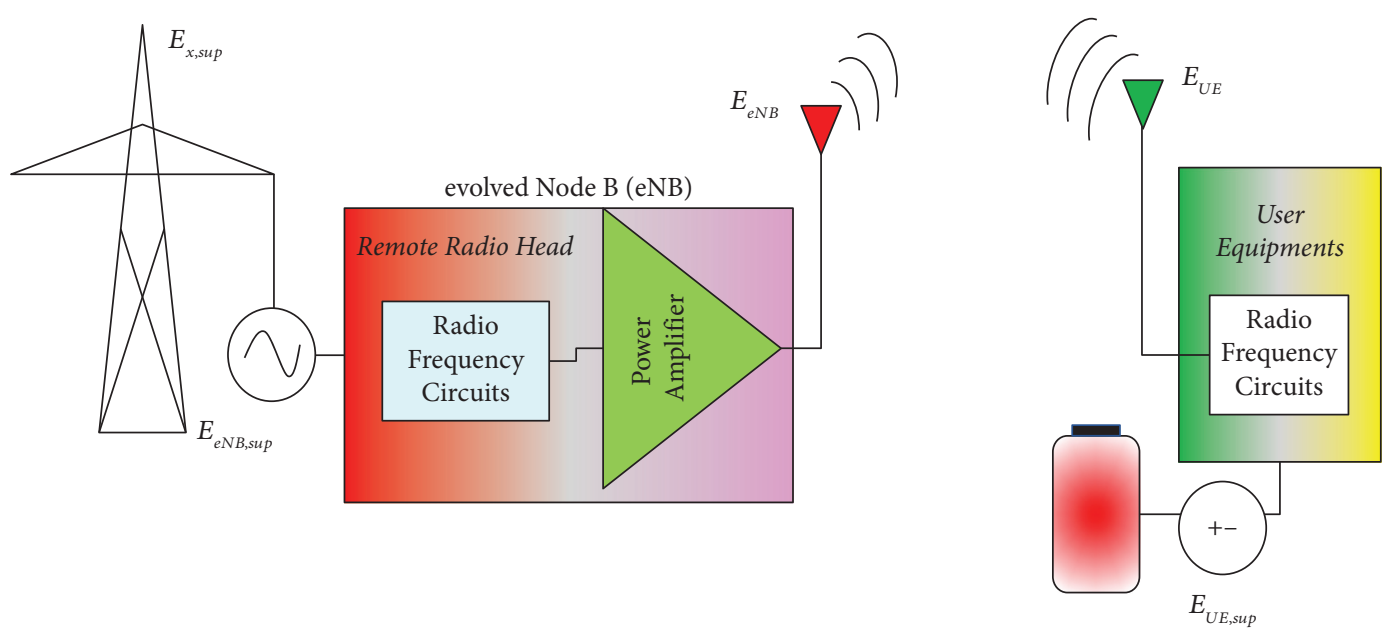

Figure 1: A simple picturization of the evolved node B and the user equipments that are included in the power model.

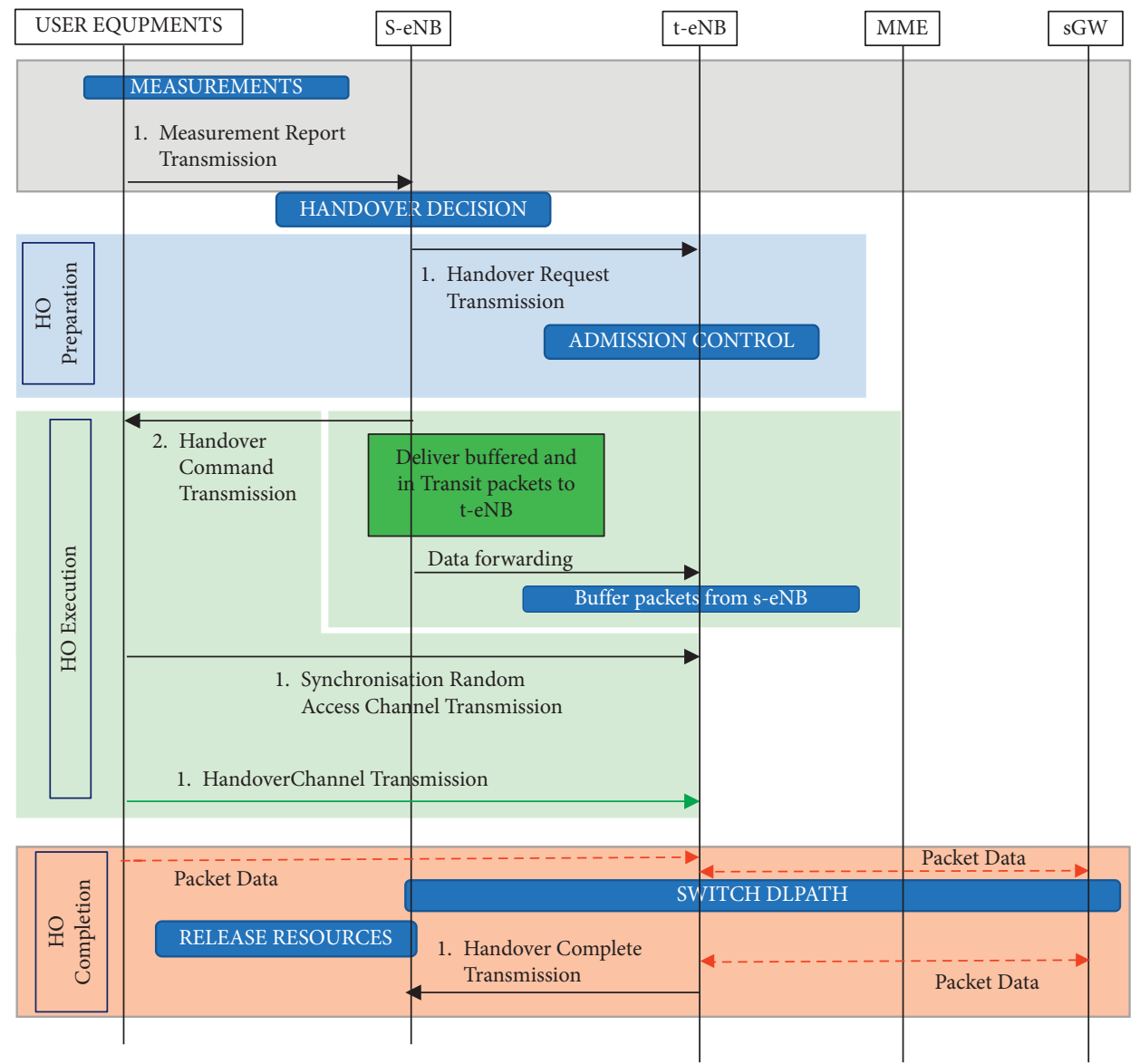

Figure 2: Mobility management entity/security gateway handover procedure [8].

where $M_{\mathrm{RB}}^{\mathrm{DL}}$ is the numerical count of the resource segments in the DownLink represented as DL in a system with bandwidth $B$ Width ${ }_{\text {sys }}$.

Allocated transmitted power measured in Watts in each message is expressed as

$$
E_{x}^{s}=E_{x}^{\mathrm{RB}} \cdot M_{\mathrm{RB}}^{s},
$$

where $x=\{e \mathrm{NB}, \mathrm{UE}\}$ and the number of Downlink and Uplink messages in $M_{\mathrm{RB}}^{s}$.

In particular, e-NB transmitting signalling, $E_{e \mathrm{NB}, \text { sup }}^{s}$, can be written as follows:

$$
E_{e \mathrm{NB}, \mathrm{sup}}^{s}=\frac{E_{e \mathrm{NB}}^{s}}{\eta}+\frac{M_{\mathrm{RB}}^{s}}{M_{\mathrm{RB}}^{\mathrm{DL}}}
$$


TABle 1: Power usage data.

\begin{tabular}{lc}
\hline Size of messages & $\begin{array}{c}L_{\text {HandOver Command }}=312 \text { bits } \\
L_{\text {HandOver Confirm }}=96 \text { bits }\end{array}$ \\
\hline Bits carried in a resource block & $L_{\text {Resource Block }}=176$ bits \\
\hline Efficiency of the power amplifier used & $\eta=0.423(42.3 \%)$ \\
\hline Radio frequency power supply & $E_{\mathrm{RF}, e N B}=13.3$ Watts, $E_{\mathrm{RF}, \mathrm{UE}}=3.24 \mathrm{Watts}$ \\
\hline Message transfer periods & $T_{e \mathrm{NB}}^{\text {Hand }}$ Cremmand $_{\text {Comm }}=0.5$ milliseconds \\
& $T_{\text {User Equipment }}^{\text {Hand }}=0.5$ milliseconds \\
\hline
\end{tabular}

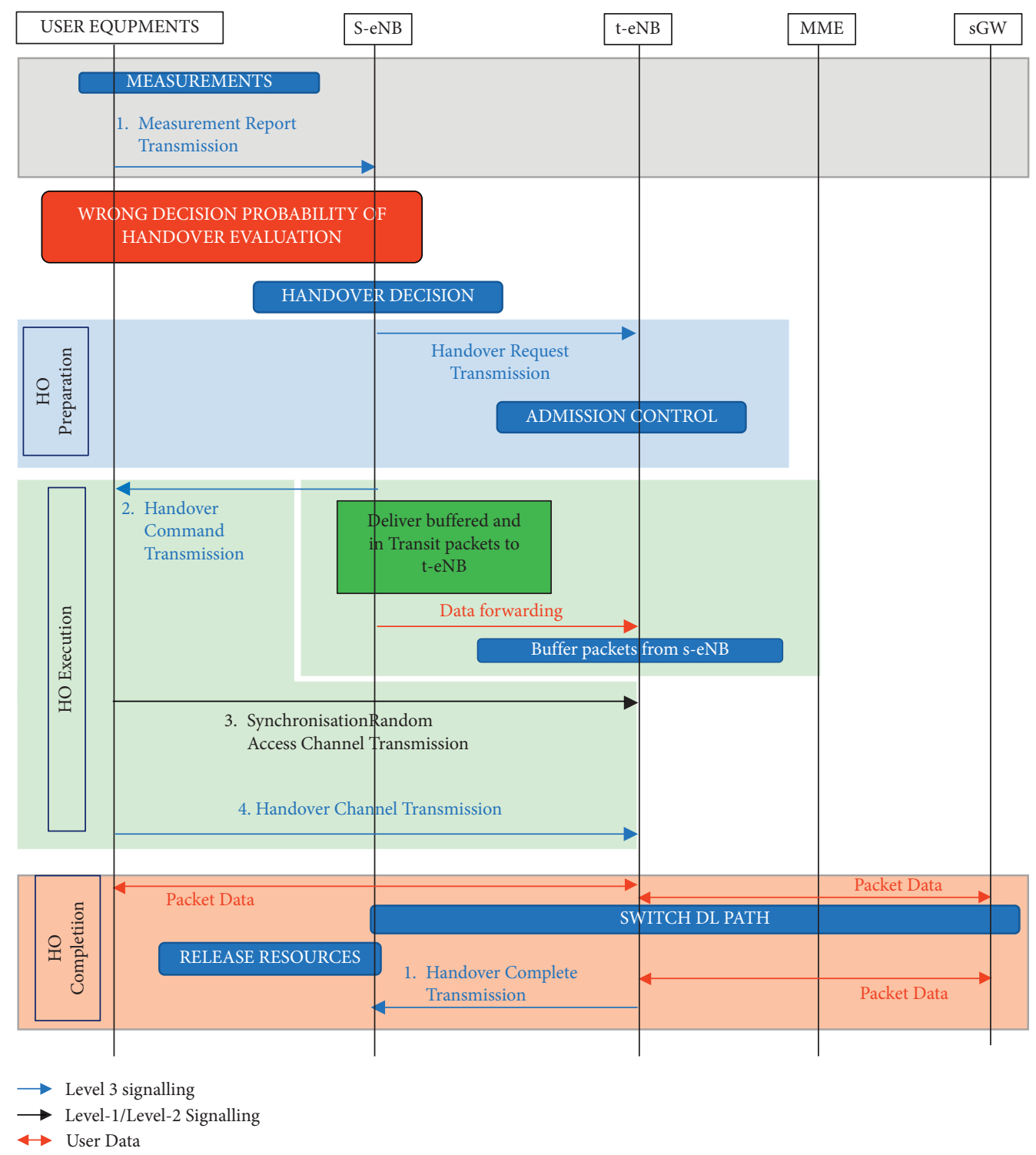

Figure 3: Proposed framework for mobility management/security gateway protocol including wrong decision probability of handover evaluation following measurements' procedure.

where $E_{e \mathrm{NB}}^{s}$ is the output transmitted power given by equation (3).

Here, time-mean supply power is

$$
\bar{E}_{x, \text { sup }}^{s}=E_{x, \sup }^{s}\left(\frac{\Delta t_{x}^{s}}{\Delta T}\right),
$$

where $E_{x, \text { sup }}^{s}$ in which $x=\{e \mathrm{NB}, \mathrm{UE}\}$ is the supply power represented in equations (3) and (4) and $\left(\Delta t_{x}^{s} / \Delta T\right)$ expresses the total duty cycle or percentage of time where the signalling is actually transmitted; we can rewrite it as

$$
\bar{E}_{x, \text { sup }}^{s}=E_{x, \text { sup }}^{s} \cdot T_{x}^{s} \cdot\left(\frac{M_{s}}{\Delta T}\right) \text {. }
$$




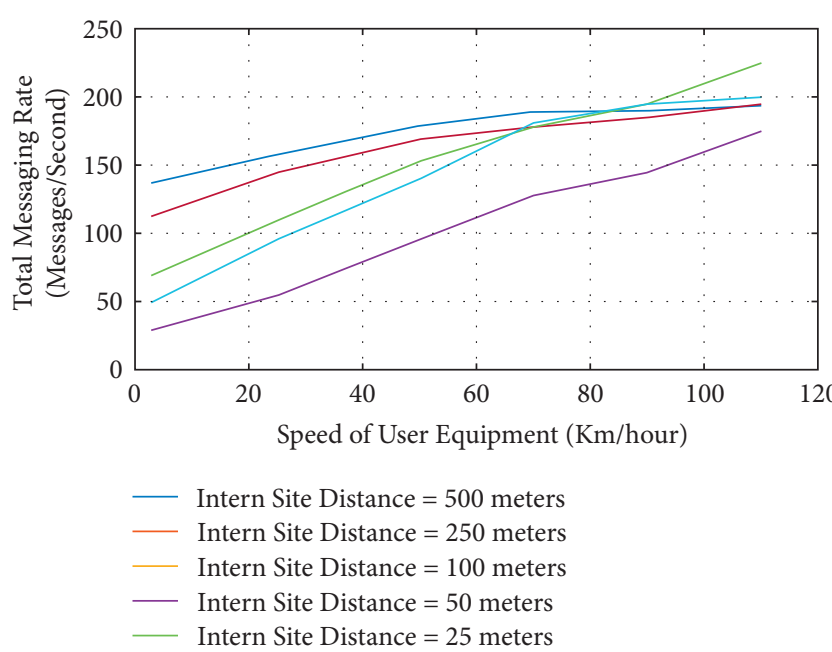

FIGURE 4: Variation of the messaging rate with the variation of speed of user equipment.

$\left(M_{s} / \Delta T\right)$ is referred as the messaging rate.

Table 1 represents the power usage data.

3.2. Proposed Solution. In this section, we combine the concept of missing handovers and unnecessary handovers with $\bar{E}_{x \text {,sup }}^{s}$, the supply power at the user equipments or at the evolved Node B (e-NB). By this procedure, we are able to find out how much the energy varied.

Generally, in conventional handover initiation procedure in long-term evolution networks or LTE networks, measurements are conducted, and then, after that, the handover request transmission starts from the end of the e-NB that is supporting the user equipment.

Now, we propose a new framework that will first evaluate the wrong decision probability of handover procedure following the technique proposed in Section 4 based on the measurement report that has been generated.

We will measure the wrong decision probability of the handover procedure by equation (6) presented below:

$$
P_{\text {wrong }}=P_{\text {missing_HandOver }}+P_{\text {unnecessary_HandOver }} \text {. }
$$

After the evaluation of the probability value $P_{\text {wrong }}$, we would relate it to the power consumption value presented via equation (5) in this section.

After doing this, we obtained the result presented in Figure 3, where we found that compared to the conventional procedure presented in Figure 1 by following the new methodology (see Figure 3) of first evaluating the wrong decision probability and then including that the value (as evaluated using equation (6)) in the handover decision process reduces the energy consumed in the handover process by avoiding unnecessary handovers in the network.

\section{Results and Discussion}

This section contains numerical data on the signalling rate during the $\mathrm{HO}$ procedure. The influence of intersite distance (ISD) and UE speed on an aggregate signalling rate is

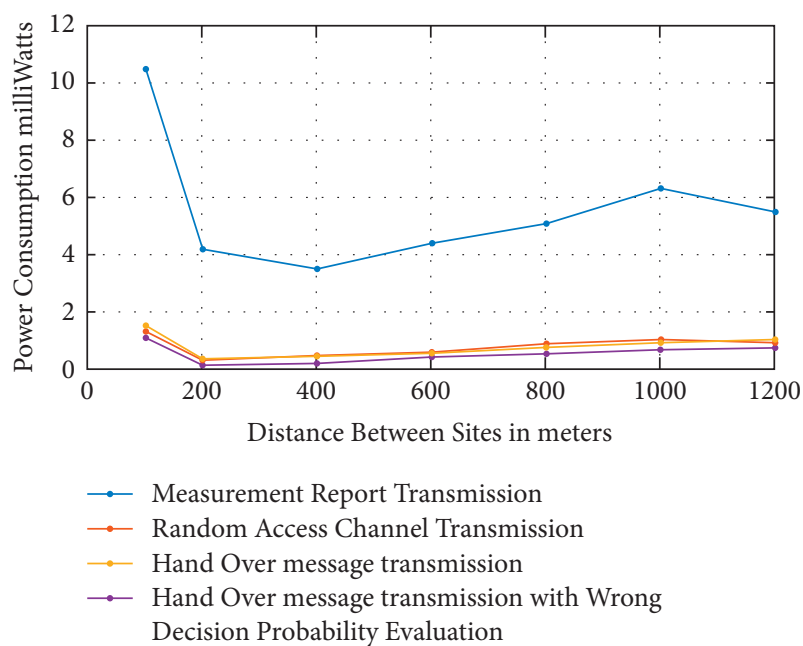

FIGURE 5: Evaluation of power consumption (in milliwatts) with the variation of distance between sites of base station for (a) transmission of measurement report, (b) random access channel transmission, (c) handover message transmission, and (d) handover message transmission with the evaluation of wrong decision probability.

depicted in Figure 4. This is the issue because higher UE speeds for microcells cause movement away from the source cell, which might cause problems during $\mathrm{HO}$ and hence increase signalling overhead.

The average supply power consumption as a result of the numerous HO signalling broadcasts is calculated in this section. The values for constant speed, offset, and TTT are shown in Figure 5. Because we can eliminate the UL impairment problems that arise for small and large ISDs, we can attain the lowest power consumption for ISD $500 \mathrm{~m}$, which is under the simulated assumptions provided.

According to Figures 4 and 5, the performance of the power consumption and the speed of UEs is analyzed for investigating the performance to handover. In this work, the handover by means of mobility management and security gateway protocol achieves the better performance.

\section{Conclusion and Future Scope}

To obtain the simple picturization of the evolved node $B$ and the user equipments that are included in the power model has been discussed effectively. The proposed framework for mobility management/security gateway protocol including wrong decision probability of hand over evaluation following measurements' procedure has designed successfully. The power consumption of $\mathrm{HO}$ command transmission (DL/eNB power consumption) is significantly higher as compared to others (UL/UE power consumption). The lowest ISD has the largest power usage, as can be seen in both graphs. Result of the handover from the enodeB to the user equipment is 0.5 milliseconds. It is possible to transport messages in a reasonable amount of time. The massage transfer durations will be extended in the near future. 


\section{Data Availability}

The datasets used and/or analyzed during the current study are available from the corresponding author upon reasonable request.

\section{Conflicts of Interest}

The authors declare that they have no conflicts of interest.

\section{References}

[1] N. Nasser, A. Hasswa, and H. Hassanein, "Handoffs in fourth generation heterogeneous networks," IEEE Communications Magazine, vol. 44, no. 10, pp. 96-103, 2006.

[2] A. Hasswa, N. Nasser, and H. Hassanein, "A seamless contextaware architecture for fourth generation wireless networks," Wireless Personal Communications, vol. 43, no. 3, pp. 10351049, 2007.

[3] P. Pawar, K. Wac, B. J. Van Beijnum, P. Maret, A. van Halteren, and H. Hermens, "Context-aware middleware architecture for vertical handover support to multihomed nomadic mobile services," in Proceedings of the 2008 ACM symposium on Applied Computing, pp. 481-488, ACM, New York, NY, USA, March 2008.

[4] S. Dekleva, J. P. Shim, U. Varshney, and G. Knoerzer, "Evolution and emerging issues in mobile wireless networks," Communications of the ACM, vol. 50, no. 6, pp. 38-43, 2007.

[5] M. Ghaderi and R. Boutaba, "Call admission control in mobile cellular networks: a comprehensive survey," Wireless Communications and Mobile Computing, vol. 6, no. 1, pp. 69-93, 2006.

[6] D. J. Deng and H. C. Yen, "Quality-of-service provisioning system for multimedia transmission in IEEE 802.11 wireless LANs," IEEE Journal on Selected Areas in Communications, vol. 23, no. 6, pp. 1240-1252, 2005.

[7] E. Stevens-Navarro and V. W. S. Wong, "Comparison between vertical handoff decision algorithms for heterogeneous wireless networks," in Proceedings of the 2006 IEEE 63rd Vehicular Technology Conference, IEEE, Melbourne, VIC, Australia, May 2006.

[8] A. Singhrova and N. Prakash, "A review of vertical handoff decision algorithm in heterogeneous networks," in Proceedings of the 4th International Conference on Mobile Technology, Applications, and Systems and the 1st International Symposium on Computer Human Interaction in Mobile Technology, pp. 68-71, ACM, Singapore, September 2007.

[9] I. Joe and S. Hong, "A mobility-based prediction algorithm for vertical handover in hybrid wireless network," in Proceedings of the 2007 2nd IEEE/IFIP International Workshop on Broadband Convergence Networks, Munich, Germany, May 2010.

[10] A. Singhrova and N. Prakash, "Vertical handoff decision algorithm for improved quality of service in heterogeneous wireless networks," IET Communications, vol. 6, no. 2, pp. 211-223, 2012.

[11] R. Tamijetchelvy, G. Sivaradji, and P. Sankaranarayanan, "Dynamic MAPT approach for vertical handover optimization in heterogeneousnetwork for CBR and VBR QoS guarantees," in Proceedings of the International Conference on Information and Communication Technologies (ICICT), pp. 1164-1172, Karachi, Pakistan, December 2015.

[12] J. McNair and F. Zhu, "Vertical handoffs in fourth-generation multinetwork environments," IEEE Wireless Communications, vol. 11, no. 3, pp. 8-15, 2004.
[13] W. T. Chen, J. C. Liu, and H. K. Huang, "An adaptive scheme for vertical handoff in wireless overlay networks," in Proceedings of the Tenth International Conference on Parallel and Distributed Systems, 2004, pp. 541-548, IEEE, Newport Beach, CA, USA, July 2004.

[14] F. Zhu and J. McNair, "Optimizations for vertical handoff decision algorithms," in Proceedings of the Wireless Communications and Networking Conference, 2004, pp. 867-872, IEEE, Atlanta, GA, USA, March 2004.

[15] W. T. Chen and Y. Y. Shu, "Active application oriented vertical handoff in next-generation wireless networks," in Proceedings of the IEEE Wireless Communications and Networking Conference, 2005, pp. 1383-1388, IEEE, New Orleans, LA, USA, March 2005.

[16] W. Zhang, "Handover decision using fuzzy MADM in heterogeneous networks," in Proceedings of the Wireless Communications and Networking Conference, 2004, pp. 653-658, IEEE, Atlanta, GA, USA, March 2004.

[17] Q. Song and A. Jamalipour, "A network selection mechanism for next generation networks," in Proceedings of the IEEE International Conference on Communications, 2005, pp. 1418-1422, IEEE, Seoul, Republic of Korea, May 2005.

[18] M. Peng, "System architecture and key technologies for 5G heterogeneous cloud radio access networks," IEEE Network, vol. 29, no. 2, pp. 6-14, 2015.

[19] A. Checko, "Cloud RAN for mobile networks-a technology overview," IEEE Communications Surveys and Tutorials, vol. 17, 2015.

[20] A. Alhammadi, M. Roslee, M. Y. Alias, I. Shayea, and S. Alraih, "Dynamic handover control parameters for LTE-A/ 5G mobile communications," in Proceedings of the 2018 Advances in Wireless and Optical Communications (RTUWO), pp. 39-44, IEEE, Riga, Latvia, November 2018.

[21] E. S. Lohan, M. Koivisto, O. Galinina et al., "Benefits of positioning-aided communication technology in high-frequency industrial IoT," IEEE Communications Magazine, vol. 56, no. 12, pp. 142-148, 2018.

[22] D. Gómez Depoorter and W. Kellerer, "Designing the airground data links for future air traffic control communications," IEEE Transactions on Aerospace and Electronic Systems, vol. 55, no. 1, pp. 135-146, 2019.

[23] N. Cheng, W. Xu, W. Shi et al., "Air-ground integrated mobile edge networks: architecture challenges and opportunities," IEEE Communications Magazine, vol. 56, no. 8, pp. 26-32, 2018.

[24] Z. Zhou, J. Feng, L. Tan, Y. He, and J. Gong, "An air-ground integration approach for mobile edge computing in IoT," IEEE Communications Magazine, vol. 56, no. 8, pp. 40-47, 2018.

[25] Z. Zhou, J. Feng, C. Zhang, Z. Chang, Y. Zhang, and K. M. S. Huq, "SAGECELL: software-defined space-airground integrated moving cells," IEEE Communications Magazine, vol. 56, no. 8, pp. 92-99, 2018.

[26] J. Liu, Y. Shi, Z. M. Fadlullah, and N. Kato, "Space-air-ground integrated network: a survey," IEEE Communications Surveys and Tutorials, vol. 20, no. 4, pp. 2714-2741, 2018.

[27] X. Zhou, S. Durrani, J. Guo, and H. Yanikomeroglu, "Underlay drone cell for temporary events: impact of drone height and aerial channel environments," IEEE Internet of Things Journal, vol. 6, no. 2, pp. 1704-1718, 2019.

[28] C. Zhang, W. Zhang, W. Wang, L. Yang, and W. Zhang, "Research challenges and opportunities of UAV millimeterwave communications," IEEE Wireless Communications, vol. 26, no. 1, pp. 58-62, 2019. 
[29] Z. Feng, L. Ji, Q. Zhang, and W. Li, "Spectrum management for mmwave enabled UAV swarm networks: challenges and opportunities," IEEE Communications Magazine, vol. 57, no. 1, pp. 146-153, 2019.

[30] I. García-Miranda, L. Hernán-Muñoz, F. Díaz, A. Bregon, M. A. Martinez-Prieto, and J. López-Leones, "AIRPORTS metrics: a big data application for computing flights' performance indexes based on flown trajectories," in Proceedings of the IEEE/AIAA Digital Avionics Systems Conference (DASC), pp. 1-10, London, UK, September 2018.

[31] S. Neeraja and A. Abhishiktha, "Comparison of handover probability analysis for multiple heterogeneous wireless networks," in Proceedings of the IEEE International Conference Communication and Electronics Systems (ICCES), pp. 1071-1075, Coimbatore, India, October 2018.

[32] A. K. Paul, K. Hidehiko, T. Atsuo, and H. Teruyuki, "Effect of AQM based RLC buffer management on the eNB scheduling algorithm in LTE network," Technologies, vol. 5, 2016.

[33] D. Li, J. Theunis, K. Sleurs, J. Potemans, E. H. Van Lil, and A. R. Van de Capelle, "Improving RED performance during handovers in wireless IP networks," in Proceedings of the 2007 4th International Symposium on Wireless Communication Systems, pp. 441-445, Trondheim, Norway, October 2007.

[34] J. Moysen, F. Ahmed, M. García-Lozano, and J. Niemelä, "Unsupervised learning for detection of mobility related anomalies in commercial LTE networks," in Proceedings of the 2020 European Conference on Networks and Communications (EuCNC), pp. 111-115, Dubrovnik, Croatia, June 2020. 